\title{
Efficacy of Focused Stimulation in Early Language Intervention Program for Toddlers with Repaired Cleft Palate
}

\author{
M Pushpavathi*, Kavya V and Akshatha V \\ Department of Speech-Language Pathology, All India Institute of Speech and Hearing, India
}

Submission: July 04, 2017; Published: July 18, 2017

*Corresponding author: M Pushpavathi, Department of Speech-Language Pathology, All India Institute of Speech and Hearing (AIISH), Manasagangothri, Mysuru - 570 006, India, Tel: 0821-2502570; E-mail: shivanna.pushpa@gmail.com

Abstract

Early language intervention is required soon after the surgery for children with cleft lip and palate (CLP) and extensive research has documented the usefulness of the same. The present study attempts to provide an insight on the efficacy of speech and language therapy using focused stimulation approach by involving mothers. The different parameters such as phonemic inventory and vocabulary of the toddlers were investigated before and after 20 sessions of speech and language therapy. Six toddlers with repaired cleft lip and palate between the age ranges of 2-3 years served as participants for the program. The speech and language baseline was established by measuring vowel, consonant inventory, proto-words and true words of the toddlers. Speech and language therapy was given for 20 sessions. Mothers were oriented and counseled for speech stimulation. A post-therapy measurement was done after 20 sessions to analyze the speech behaviors of the toddlers.

A non-parametric test was carried out due to the small Sample size. Wilcoxon Signed Rank test was administered which highlighted that parameters such as the number of phonemes, vowels, consonants showed statistically significant difference $(\mathrm{p} \leq 0.05)$ between the pretest and posttest measures. Also phonemes such as high and mid vowels, bilabials and fricatives showed statistically significant difference ( $p \leq 0.05)$. The results indicated an improvement in the type and number of vowels, consonants and true words among the toddlers. There was also a reduction in the non-verbal behaviors in the toddlers post-therapy. The results highlight the importance of involving parents and efficacy of early language intervention program.

Keywords: Cleft palate; Early Intervention Program; Focused Stimulation; Phonemic Inventory; Vocabulary

Abbreviations: CLP: Cleft lip and palate; EMT: Enhanced Milieu Teaching; FS: Focused Stimulation; TD: Typically Developing; IPA: International Phonetic Alphabet

\section{Introduction}

Cleft lip and palate (CLP) is a congenital deformity which hinders effective communication in early childhood due to associated speech and language disorders. Children with CLP demonstrate multiple problems such as dental problems, psychological problems which may result in a negative impact on the child's social, emotional and educational well being [1-4]. The birth rate of CLP in India is reported to be 1.09 for every 1000 live births [5]. A more recent statistical survey shows that the incidence has risen to 7 per 1,000 children, and as a result, CLP is declared as one of the most common deformities seen among Indian children [6]. It is also reported that nearly 40,000 children are born in India every year with CLP [6].
Over the last two decades, different studies have highlighted the associated problems seen in speech of children with CLP such as limited sound inventory, expressive language delay, limited vocabulary, reduced communication attempts, hypernasality, nasal air emission, reduced speech intelligibility and compensatory articulation $[7,8]$. These multiple associated problems need to be dealt by a team of experts from birth to the age of 18 years. Extensive research has documented that early language intervention is required soon after the surgery for children with CLP and it has been proven that such language intervention programs have been effective in facilitating both language and speech in such children [9]. 


\section{Global Journal of Otolaryngology}

Traditionally, the early intervention for children with CLP focused mainly on aspects related to speech production such as articulatory skills, resonance abilities and intelligibility. Studies and reviews carried out in recent times have highlighted that children with CLP obtain considerably poorer scores in the language domain especially in expressive abilities, when compared to their typically developing peers [8]. This underlines the fact that focus should also be brought to improve linguistic skills during the early stages of intervention in children with CLP.

As far as treatment approaches is concerned, there is no common consensus among SLP's across the world and different techniques and programs are being followed. Over the last two decades, various hybrid models such as Enhanced Milieu Teaching (EMT) and Focused Stimulation (FS) approach have been widely used for improving linguistic skills in children with CLP [9]. These models advocate promoting of language in natural conversation. The EMT approach prompts functional language use in activities of daily living by applying the behavioral principles [10]. The Focused Stimulation approach is also based on the naturalistic intervention model [11-13]. This approach lays emphasis on techniques involving modeling and responsive interaction. The environmental arrangement and activities are organized in such a way that functional communication is taught to the child. This model assumes that language is learnt in a meaningful interactive setup with conversational partners who promote vocabulary building of the child [9].

A recent meta-analytic study was carried out wherein an indepth review of journal articles was systematically searched and complied from 1970 till 2011 [14]. They studied the efficacy of early language intervention in children with CLP in the age range of 1.6 to 4.11 years. The authors reported that the results of all the studies opined that an active participation of parents in the intervention sessions lead to better communicative abilities in the children, by improving the linguistic abilities of the children with CLP. The study also brought to light the fact there is a dearth of studies providing empirical support for early intervention in the CLP population and the involvement of parents in the same. Thus some relatively widespread research in the western context on early parent-implemented intervention has documented the efficacy of the same.

In the Indian scenario, limited studies have been carried out highlighting the relationship between early intervention and the speech and language output of children with CLP. Also there is a dearth of studies in India which investigate the effectiveness of a structured parent-implemented program for young children with CLP. One such study investigated the nature of stimulation provided by mothers of typically developing (TD) children in the age range of 3-5 years [15]. Mohite's Home Environment Inventory [16] was administered before and after the intervention sessions in order to judge the stimulation provided by mothers. The mothers in the experimental group were exposed to training for 16 weeks while the control group was not exposed to any kind of program. In post-intervention the results indicated that mothers in the experimental group were significantly better in providing stimulation to their children when compared to their counterparts [15].

Another study was conducted recently wherein the pattern of early speech and language development in children with unrepaired cleft of palate (clinical group) was studied by comparing them with age and gender matched typically developing (TD) children (non-clinical group) [17]. A total of 1000 utterances (100 from each child) obtained through video recordings were transcribed and the distribution of phonemes, syllabic and linguistic structures between clinical and agematched non-clinical groups were analyzed. The findings revealed that the vowel repertoire of toddlers with unrepaired cleft of palate showed a greater incidence of back and open vowels whereas TD children showed a greater frequency of close vowels. With respect to consonants it was observed that the clinical group produced phonemes which were mostly confined to either extremes of the vocal tract such as glottals and bilabials. Also stops and nasals were seen to occur frequently with respect to manner of articulation which could be attributed to the structural limitations. The consonant repertoire of TD children showed greater variety compared to children with CLP. The examination of syllabic structures showed that clinical group produced fewer polysyllables compared to the non-clinical group and were restricted to VCV syllables. The clinical group evinced a slight delay in expressive language of 2-6 months, but this was not statistically significant. The study thereby highlights the difference in early speech and linguistic characteristics between clinical and non-clinical group, thereby stressing on the need for early intervention in this population.

A case study was conducted along similar lines recently, wherein the effectiveness of a systematic focused stimulation approach was assessed on a toddler with expressive language delay secondary to repaired cleft of palate (RCP) [18]. The results revealed that post-intervention, there were significant changes in the phoneme repertoire and vocabulary of the child. An increase in the frequency of speech sounds, for both vowels and consonants as well as for true words was observed. These findings yet again illustrate the positive effects of a structured and parent-implemented intervention program for children with CLP especially during the formative years.

Although several approaches have been proposed in the western context to improve the language abilities of children with CLP, very few studies in the Indian context have focused on investigating the long term effects of language intervention strategies in this group. Hence, the present study attempts to provide an insight on the efficacy of speech and language therapy using focused stimulation approach by involving mothers and also aims to describe the speech and language characteristics of toddlers with CLP before and after speech-language intervention. 
The objectives of the present study are as follows:

a) To study the impact of parent-implemented intervention program on speech output of toddlers with CLP with respect to parameters such as frequency of occurrence of vowels and consonants before and after the intervention program.

\section{Method}

\section{Participants}

Table 1: Details of the participants of the study.

\begin{tabular}{|c|c|c|c|c|c|}
\hline \multirow[t]{2}{*}{ Participants } & \multirow[t]{2}{*}{ Age of surgery } & \multirow{2}{*}{$\begin{array}{l}\text { Age of Enrollment } \\
\text { for therapy }\end{array}$} & \multicolumn{2}{|c|}{ Language age } & \multirow{2}{*}{$\begin{array}{c}\text { Syllabic structure } \\
\text { (Baseline) }\end{array}$} \\
\hline & & & RLA & ELA & \\
\hline 1 & 1 year (Palate) & 1.6 years & 16-18 months & 9-10 months & CV, VCV (non-meaningful utterances) \\
\hline 2 & $\begin{array}{c}5 \text { months (lip) \& } 1 \text { year } \\
\text { (Palate) }\end{array}$ & 3 years & 33-36 months & 20-22 months & CV,VCV,CVCV (1-2 word utterances) \\
\hline 3 & 1.6 years (Palate) & 2.6 years & 27-30 months & 16-18 months & CV,VCV,CVCV(non- meaningful utterances) \\
\hline 4 & 1 year (Palate) & 2 years & $22-24$ months & 9-10 months & CV, VCV (non-meaningful utterances) \\
\hline 5 & $\begin{array}{c}7 \text { months (lip) \&1.6 yrs } \\
\text { (palate) }\end{array}$ & 2 years & $22-24$ months & $18-20$ months & CV,VCV,CVCV (one-word utterances) \\
\hline 6 & 1.8 years & 2.6 years & 27-30 months & 12-14 months & CV,VCV (non- meaningful utterances) \\
\hline
\end{tabular}

Six non-syndromic toddlers in the age range of 2-3 years who were diagnosed to have Expressive Language Delay secondary to repaired cleft lip and palate served as subjects for the study. All the participants had Kannada[a] as their native language. The speech and language evaluation was carried out by a qualified Speech Language Pathologist using Receptive Expressive Emergent Language Scale [19]. The children had normal auditory and cognitive abilities. The participants were enrolled for the Early Language Intervention Programme (ELIP) at the Unit for Structural and Oro-facial Anomalies, All India Institute of Speech and Hearing (AIISH). An informed consent (approved by the AIISH Ethical Committee) was obtained from the parents prior to their participation in the study. The details of the subjects are shown above (Table 1).

\section{Note:}

[a] Kannada language is a Southern Dravidian language and is one of the oldest living languages in the world. It consists of 6 swaras (vowels) and 24 vyanjanas (consonants) excluding the aspirated counterparts. It has a simple syllabic structure consisting of V, CV and CCV combinations. In Kannada language consonants do not occur in word-final position and CV syllables form the major portion of the syllabic structure.

\section{Enrollment to the Early Language Intervention Programme}

After the participants were enrolled for the Early Language Intervention Programme, a pre-orientation questionnaire regarding 'Awareness of parents on issues related to cleft lip and palate' [20] was administered on the mothers. This questionnaire was administered to assess the levels of awareness present in them on various aspects related to CLP. Following this, a detailed b) To investigate the effect of early language intervention true words, prototype words and non meaningful utterances before and after the intervention program. on linguistic parameters of toddlers such as frequency of orientation was provided to them regarding the incidence, social stigma, complications, associated problems and management of children with CLP.

\section{Data collection}

A detailed pre-therapeutic assessment was carried out to establish the speech and language skills present in the child prior to attending therapy and the baseline was established by measuring the vowel and consonant inventory, proto words and true words of the toddlers. Audio-video recording was carried out using a Handycam recorder (Sony DCR-SR88) for each child during the initial sessions in order to establish the baseline of each child prior to speech-language therapy.

The recordings were carried out in a quiet room during unstructured and free play sessions between the mother and the child. Each recording was done for an approximate duration of 45-50 minutes when the child was co-operative. In instances wherein the child was not co-operative various strategies such as a few trial recordings were carried out or environmental modifications such as placing the handycam in a hidden way or giving breaks in between sessions were done. The mothers were oriented and counseled for speech stimulation. Following this, a total number of 20 therapy sessions for each child were conducted by the Speech Language Pathologist (SLP), wherein the mother was also an active participant. The handycam recorder was placed on a tripod stand at a distance of approximately 8-10 feet from the child in such way that the entire play area was captured and so that the child was not distracted. The recording was done during the baseline session and after the $20^{\text {th }}$ session of speech and language interaction. Thus 2 recordings were done for each mother-child dyad and in total 12 video recordings were available for each child. The data was further analyzed. 


\section{Speech and language intervention}

The speech-language therapeutic sessions were carried out in a clinical setup by a qualified and experienced SLP and each session lasted for forty-five minutes. A master lesson plan was prepared for each child by taking up specific goals and activities, based on the baseline which was ascertained during the pretherapeutic assessment. Goals with respect to resonance such as increasing the oral airflow and improving the oro motor abilities, speech-related goals focusing on increasing the consonant inventory, along with this the linguistic goals such as increasing the functional and lexical vocabulary were also considered. An interactive play way method was used to improve vocabulary, vowel and consonant inventory and to increase the frequency of meaningful utterances.

Focused stimulation approach was demonstrated to the mother during therapeutic sessions using low-cost materials and commonly available toys. A multisensory approach was used to increase the oromotor movements. Visual feedback using mirror work and tactile cues with interesting oromotor activities was provided appropriately wherever required. A corpus of vocabulary was prepared in the master lesson plan which contained the most commonly used functional words by toddlers. This was done with the aim of increasing the child's functional vocabulary. The positive behaviors and behaviors which were attempted correctly by the child were reinforced with token and tangible reinforcements to improve their cooperation and confidence in doing the activity. The mother was told to carry out a similar training program at home in the absence of direct supervision of the SLP.

\section{Data Analysis}

Each of the video and audio-taped session of mother-child interaction during unstructured play was transcribed using International Phonetic Alphabet (IPA) symbols and analyzed. With respect to the toddlers, the frequency of occurrence of phonemes, i.e. vowels and consonants were calculated. Vowels were classified as high (/i/), mid (/e/, /æ/) and low (/a/) based on the tongue height and their frequencies were calculated. The frequency of occurrence of different types of consonants was calculated based on the place and manner of articulation. Also the total number of true words and proto words were obtained and tabulated. The consistency of the true words produced was also noted and a target word which was produced twice or more accurately out of five times was considered to be consistent. The multiple recordings mentioned above were thereby carried out to obtain a consistent corpus of utterances. A Pretest-Posttest design was used in order to monitor the changes across the baseline and the $20^{\text {th }}$ session.

\section{Results and Discussion}

The present study is one of the preliminary attempts to highlight the importance of a parent-implemented speech and language intervention program in toddlers with CLP, by examining the changes in the child's speech and language inventory before and after intervention. The Pretest-Posttest measures were compared for the speech and linguistic parameters of toddlers and the findings obtained have been highlighted below.

\section{Effect of intervention on speech and language output of toddlers}

The effect of early intervention on speech related measures of toddlers were analyzed by calculating the frequency of occurrence of vowels based on their tongue height. The frequency of occurrence of consonants was calculated based on the place of articulation (bilabials, dentals, alveolars, labiodentals, velars and glottals) and manner of articulation (nasals, stops, fricatives, glides and liquids).

\section{Speech related measures}

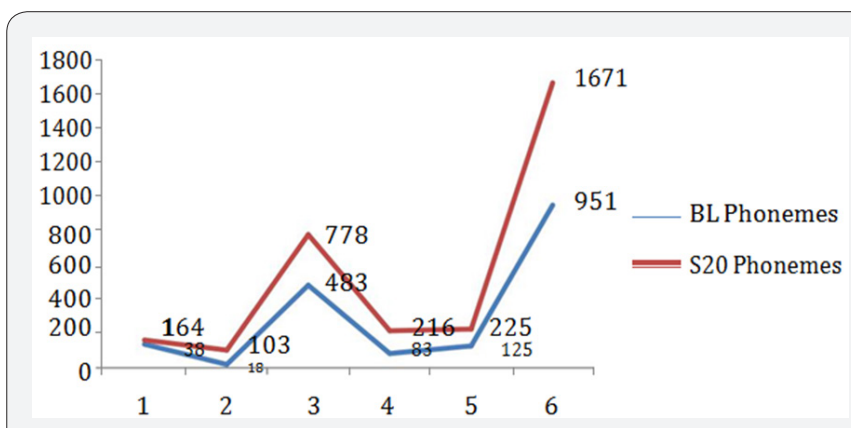

Figure 1: Frequency of occurrence of phonemes across six participants and sessions (BL-Baseline; $\mathrm{S} 20-20^{\text {th }}$ session).

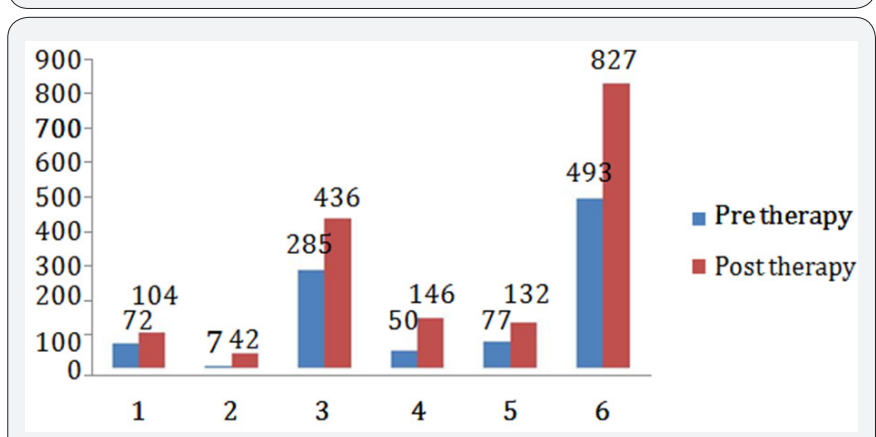

Figure 2: Frequency of occurrence of vowels across six participants in pre- and post-therapy conditions.

The frequency of occurrence of phonemes which is the total number of vowels and consonants across participants and sessions were obtained and analyzed. The aboveline graph (Figure 1) shows the frequency of total phonemes which includes both the vowels and consonants, with reference to baseline and the $20^{\text {th }}$ session. The graph depicts a trend wherein a clear difference is seen between the total number of phonemes in the baseline and the total frequency in the $20^{\text {th }}$ session. Although the trend seen was not uniform across subjects, two of the participants showed a significant difference post-intervention whereas the others also showed a difference though not significant. This lack of uniformity in the improvement could be attributed to the nonhomogenous group wherein parameters such as chronological 


\section{Global Journal of Otolaryngology}

age and language age could not be controlled. During statistical analysis, Wilcoxon Signed Rank test highlighted that certain phonemes showed a statistically significant difference $\left({ }^{*} \mathrm{p} \leq 0.05\right)$ between the pretest and posttest measures which is discussed below.

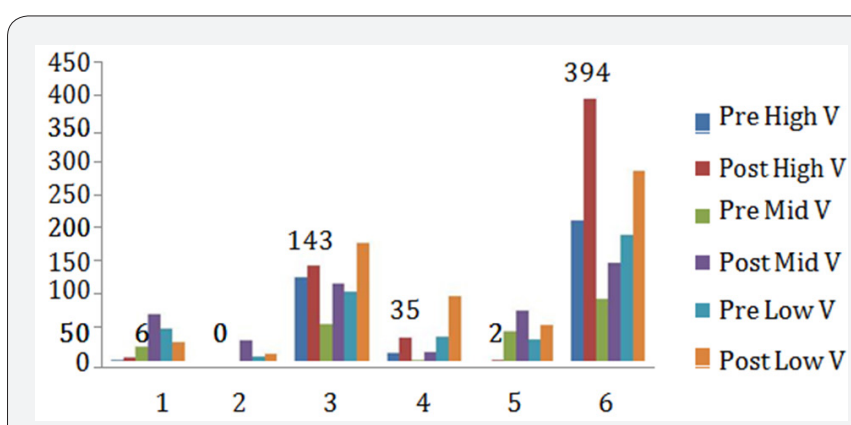

Figure 3: Distribution of high, mid and low vowels across the six participants in the pre and post-therapy conditions.

Table 2: Statistical analysis of vowels comparing pre and post-test conditions $\left({ }^{*} p \leq 0.05\right)$

\begin{tabular}{|c|c|c|c|c|c|}
\hline Vowels & \multicolumn{2}{|c|}{ Mean } & \multicolumn{2}{c|}{ S.D. } & p value \\
\hline & Pre & Post & Pre & Post & \\
\hline High & 58.50 & 96.66 & 89.45 & 155.49 & $0.043^{*}$ \\
\hline Mid & 36.00 & 75.66 & 35.80 & 50.62 & $0.027^{*}$ \\
\hline Low & 69.50 & 108.83 & 66.78 & 104.80 & 0.075 \\
\hline
\end{tabular}

The bar graphs above shows the frequency of vowels with respect to tongue height in the pre-and post-therapy conditions. The graphs clearly indicate a trend wherein all the vowels showed an increase in the $20^{\text {th }}$ session compared to the baseline. Statistical analysis indicated that certain vowels such as high and mid vowels showed a statistically significant difference $\left({ }^{*} p \leq 0.05\right)$ between the pre and posttest measures which is depicted in Table 2. The results thus indicated an improvement in the number of vowels among the toddlers.

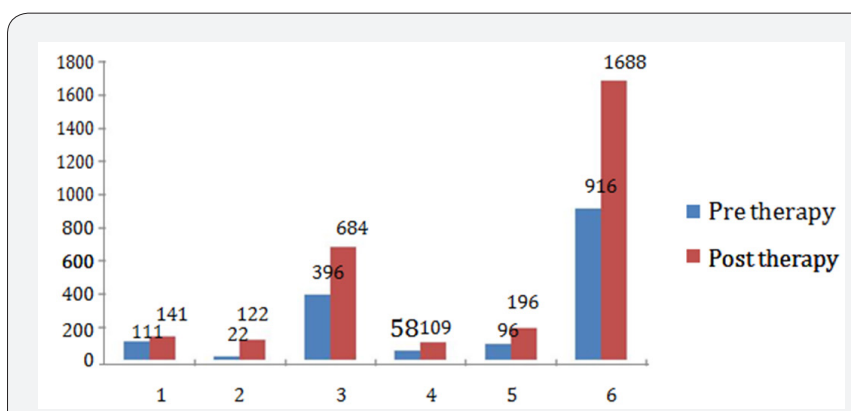

Figure 4: Frequency of occurrence of consonants across participants in pre- and post-therapy conditions.

The above Figure 4 illustrates the frequency of consonants in each of the six participants in pre-therapy and post-therapy conditions. Various consonants such as bilabials, dentals, alveolars, labiodentals, fricatives and velar sounds were present in the children's inventory. Wilcoxon Signed Rank test was administered which highlighted that certain consonants such as bilabials, fricatives and glides showed a statistically significant difference $\left({ }^{*} \mathrm{p} \leq 0.05\right)$ between the pretest and posttest measures as shown in Table 3 . With respect to place of articulation, bilabials and dentals were found to be the most frequently occurring compared to the other consonants. With respect to manner of articulation, overall, nasals showed the highest incidence followed by stops, fricatives, glides and liquids.

Table 3: Statistical analysis of consonants comparing pre and posttest conditions $\left({ }^{*} \mathrm{p} \leq 0.05\right)$.

\begin{tabular}{|c|c|c|c|c|c|}
\hline Consonants & \multicolumn{2}{|c|}{ Mean } & \multicolumn{2}{c|}{ S.D. } & P value \\
\hline & Pre & Post & Pre & Post & \\
\hline Bilabials & 49.16 & 80.16 & 34.87 & 60.44 & $0.04^{*}$ \\
\hline Dentals & 30.00 & 55.66 & 57.07 & 105.47 & 0.06 \\
\hline Alveolars & 14.66 & 29.00 & 24.87 & 46.44 & 0.06 \\
\hline Labiodentals & 4.66 & 9.17 & 7.26 & 12.30 & 0.10 \\
\hline Velars & 9.00 & 18.33 & 21.08 & 41.11 & 0.18 \\
\hline Glottals & 5.16 & 9.33 & 8.68 & 17.34 & 0.47 \\
\hline Nasals & 58.33 & 89.33 & 50.50 & 87.65 & 0.17 \\
\hline Stops & 37.83 & 58.83 & 72.88 & 110.22 & 0.07 \\
\hline Fricatives & 13.33 & 30.50 & 16.07 & 34.52 & $0.04^{*}$ \\
\hline Glides & 13.83 & 35.00 & 16.47 & 38.84 & $0.04^{*}$ \\
\hline Liquids & 9.33 & 23.83 & 14.51 & 33.81 & 0.07 \\
\hline
\end{tabular}

The results of the present study indicated that there was a noteworthy increase in the child's phoneme repertoire in terms of both vowels and consonants from baseline to post-therapy condition. In the baseline vowel inventory of the toddlers with RCLP it was clearly seen that the frequency and variety of vowels was limited and low vowels were predominant compared to high and mid vowels by a large margin. This supports the findings of a recent Indian study [17] which showed that the vowel repertoire of toddlers with CLP showed a greater incidence of back and open vowels which was attributed to structural limitations in the oral cavity. Another study along similar lines has also suggested that toddlers with CLP may follow a similar developmental pattern of vowel acquisition as that of typically developing children although there may be a delay in acquisition [21].

The analysis of the baseline consonant inventory revealed that with respect to place of articulation, bilabials and dentals showed a greater occurrence whereas with respect to manner of articulation, nasals and stops showed a higher frequency. This could be attributed to the ease of production of these phonemes as well as to their visibility. These findings are fairly in consonance with the findings of another Indian study which reported that the consonant inventory of children with CLP was limited and largely consisted of glottals, bilabials, stops and nasals [17]. Studies done over the years have revealed that the reduced variety of sounds in children with CLP could be attributed to their inability to build intraoral breath pressure required for the production of pressure consonants and might result in greater frequency of nasal sounds [22]. Investigators 
of earlier studies have stated that children with CLP use compensatory articulatory mechanisms to compensate for the faulty oral nasal coupling $[17,23]$.

The post-therapy analyses revealed certain significant findings. Although there was an increase in the frequency of both vowels and consonants certain phonemes showed changes which were statistically significant. One finding worth mentioning is that among the vowels, high and mid vowels showed a statistically significant increase post-therapy. Also it was observed that although nasalization of vowels was a predominant feature in these toddlers, accuracy in production was improved posttherapy. With respect to the consonants although only bilabials, fricatives and glides showed a statistically significant increase, all the phonemes showed a considerable increase post-therapy. These results support the findings of various studies which have highlighted that a parent-implemented intervention program led to an increase in the number and accuracy of speech sound inventory and also aided in articulatory development [2,9,24,25].

Thus the findings of the present study highlight the positive changes brought about by parent-implemented focused stimulation program in the clinical settings as well as regular training carried out at home by the parents. This underlines the need for creating awareness regarding intensive early intervention in this population. Studies consisting of larger sample sizes and longitudinal studies across longer durations of intervention comparing the clinical and non-clinical group may produce conclusive results regarding the outcomes of early intervention for young children with CLP.

\section{Language related measures}

The effects of language intervention was also examined by analyzing the frequency of occurrence of linguistic measures such as true words and proto words across participants and sessions which was then statistically analyzed. The two bar graphs (Figures $5 \& 6$ ) given above depict the frequency of occurrence of true words and proto words for each of the six participants in the baseline and the $20^{\text {th }}$ session. The graphs clearly indicate a trend wherein the frequency of true words and proto words showed a creditable increase between baseline and the $20^{\text {th }}$ session, although the differences were not statistically significant. Also the amount of increase in true words is greater than that of proto words.

The above results indicate that there was a notable enhancement in each child's vocabulary in terms of both true words and proto words from baseline to post-therapy condition. During the baseline recording, four of the six children's repertoire did not consist of any true words indicating an expressive language delay. These findings are in consonance with the studies conducted earlier which have shown that children with CLP indicate a delayed development of first word and early expressive vocabulary $[7,8,25]$. These results contradict the findings of a recent study wherein toddlers with CLP demonstrated only a slight delay in expressive language when compared to the TD children which was not statistically significant [17]. This reduction in the linguistic inventory of children with CLP could be attributed to various factors such as structural and articulatory limitations, lack of stimulation at home, overindulging of the child by the family members among others.

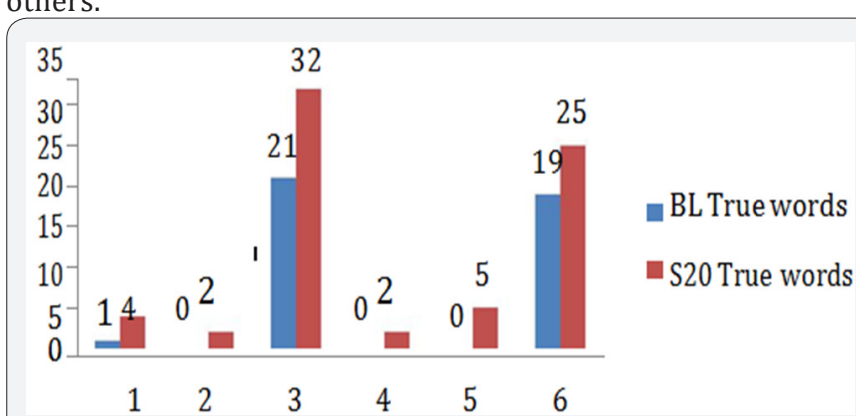

Figure 5: Frequency of occurrence of True words across six participants and sessions (BL -Baseline; $\mathrm{S} 20-20^{\text {th }}$ session).

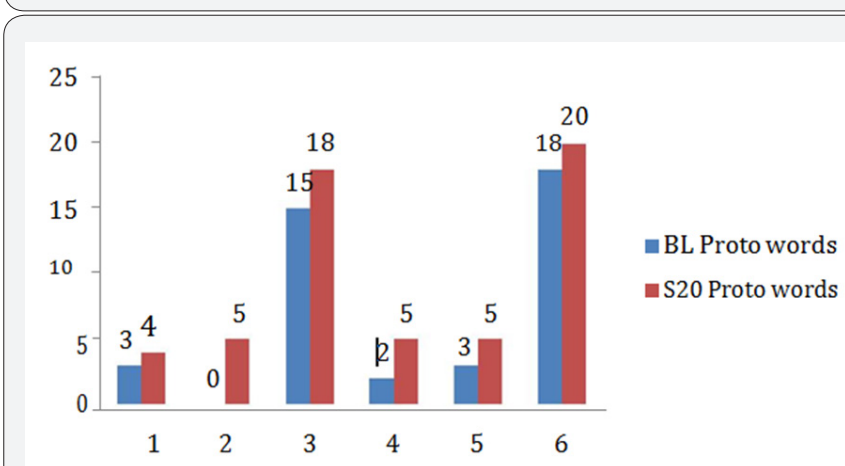

Figure 6: Frequency of occurrence of Proto words across six participants and sessions (BL -Baseline; S20 $-20^{\text {th }}$ session).

With advancement in the intervention program it was noted that there was a steady progression in the number of true words acquired by each child. By the $20^{\text {th }}$ session, all the children had acquired around twice the number of true words when compared to the baseline. Most of the children acquired functional words such as /amma/, /mama/, /anna/, /a:ne/,/avva/, /ma:mi/, / ni:ru/, /u:ta/ ${ }^{(b)}$ which emerged by the $20^{\text {th }}$ session. Amongst these true words, /amma/ was the word most frequently used by the children. The child used proto words such as /ama/ for /amba/; /mæ/ for 'sheep'; /avav/ for /bau bau/; /mamam/ for 'food'; /jiya/ for 'water'. It is also encouraging to note that children preferred the usage of true words more than proto words by the $20^{\text {th }}$ session. Thus the children acquired many true and proto words with various CVCV and VCV combinations which could be the result of continuous articulatory and linguistic stimulation by the clinician as well as the mother.

Note:

[b]/amma/-Mother; /mama/-Uncle; /anna/-Brother; / avva/-grandmother; /ma:mi/- God; /ni:ru/-Water; /u:Ta/-Food; /a:ne/-Elephant 
Another noteworthy finding emerging from the results is that the increase in true words is greater relative to the proto words. This clearly points out to the fact that children learnt to acquire a greater number of meaningful words with their production approximating the target words as the vocabulary expanded. Another significant point is that when considered from a longitudinal viewpoint, the improvement in the phonemic inventory of a child seems to have had a direct effect on the linguistic output. The greater the improvement in the frequency of vowels and consonants, the better is their progress in language in terms of true word acquisition. Although this trend has not been explored in detail in the present study it could possibly be researched in upcoming studies.

Thus the findings of the present study provide credibility to the findings that regular intervention results in gains in terms of vowels, consonants and vocabulary for children with CLP. This correlates with the findings of many studies over the years which have emphasized the effectiveness of a structured and naturalistic parent-implemented intervention program to bring about an improvement in the linguistic inventory of toddlers with CLP $[2,9,24,26]$. In the present study, Focused Stimulation approach has been used as an effective means to remediate speech and language problems in children with CLP which has also been effectively demonstrated previously [24].

\section{Conclusion}

Thus the results implicate the necessity to begin intervention at the earliest possible age in this population since adequate attention has not been paid for language acquisition in these children followed by rehabilitation. In this perspective the present study highlights the importance of regular home training and stimulation by parents in order to obtain a positive speech and language output. The study also stresses the need for involving and training the mothers of children with RCLP by trained professionals in order to bring about positive changes in the speech and language of children with CLP [27].

In India the importance and urgency of attending regular therapy and the consequences of stimulating the child during the critical years is yet to be understood by the general population. By conducting studies like the present one, it is essential to eliminate the social stigma attached to CLP and all the parameters associated with it. The findings of the present study will help parents realize the need for a parent-implemented interaction program for the child during the formative period and hopefully bring about positive changes in the outlook of parents regarding speech and language training.

\section{Acknowledgement}

This is a part of ongoing research on "Efficacy of Early Language Intervention Program for Children with Repaired Cleft lip and Palate", funded by the Department of Science and Technology (DST - No. SB/SO/HS/02/2014), Government of India. The authors would like to thank DST for funding the project. The authors would also like to thank Dr. S. R. Savithri, Director, All India Institute of Speech and Hearing, Mysuru, for providing the infrastructure to carry out the study.

\section{References}

1. Estrem T, Brown PA (1989) Early speech production of children with cleft palate. J Speech Lang Hear Res 32(1): 12-23.

2. Scherer NJ (1999) The speech and language status of toddlers with cleft lip and/or palate following early vocabulary intervention. Am J Speech Lang Pathol 8: 81-93.

3. Chapman K, Hardin-Jones M, Halter KA (2003) Relationship between early speech and later speech and language performance for children with cleft lip and palate. Clin Linguist Phon 17(3): 173-197.

4. Salas-Provance MB, Kuehn D, Marsh J (2003) Phonetic repertoire and syllable structure characteristics of 15-month-old babies with cleft palate. J Phon 31: 23-38.

5. Srinivas GR, Rajgopal RR, Bronkhorst EM, Rajendra P, Ettema AM, et al. (2001) Incidence of cleft Lip and palate in the state of Andhra Pradesh, South India. Indian J Plast Surg 4(2): 184-189.

6. Shrivatsav S (2013) Parents still don't bring cleft lip, palate kids for treatment. Times of India. Nagpur, January 18.

7. Sunitha R, Jacob M, Jacob MS, Nagarajan R (2004) Providing intervention services for communication deficits associated with cleft lip and/or palate-A retrospective analysis. APDRJ 15: 78-85.

8. DAntonio LL, Scherer NJ (2008) Communication Disorders Associated with Cleft Palate. In J Losee, R. Kirschner (Eds.), Comprehensive Cleft Care, McGraw: Hill, USA.

9. Scherer NJ, Kaiser A (2007) Early intervention in children with cleft palate. Infants Young Child 20: 355-366.

10. Kaiser AP, Hancock TB, Hester PP (1998) Parents as cointerventionists: Research on applications of naturalistic language teaching procedures. Infants Young Child 10(4): 46-55.

11. Girolametto LE (1988) Improving the social-conversational skills of developmentally delayed children. J Speech Hear Disord 53(2): 156167.

12. Girolametto LE, Pearce P, Weitzman E (1996) Interactive focused stimulation for toddlers with expressive vocabulary delays. J Speech Hear Res 39(6): 1274-1283.

13. Girolametto LE, Pearce P, Weitzman E (1997) Effects of lexical intervention on the phonology of late talkers. J Speech Hear Res 40(2): 1274-1283.

14. Meinusch M, Romonath R (2011) Early language intervention for children with cleft lip and/or palate: A systematic review. Evid Based Commun Assess Interv 5(4): 197-215.

16. Manocha A, Narang D, Balda S (2008) Role of intervention in improving maternal stimulation level. J Soc Sci 17(2): 181-184.

17. Mohite P (1989) Mohites home environment inventory: Child's observation technique. Agra: National Psychological Corporation.

18. Sreedhanya PK, Hariharan S V, Nagarajan R (2015) Early language development and phonetic repertoire in children with unrepaired cleft lip and palate: A preliminary study. J Cleft Lip Palate Craniofac Anomal 2(1): $34-40$.

19. Pushpavathi M, Kavya V, Akshatha V (2017) Efficacy of early intervention program for children with cleft lip and palate: A case study. JCLAD 5(1): 31-42. 
20. Bzoch KR, League R (1971) Assessing language skills in infancy. University Park Press Baltimore, MD, USA.

21. Thammaiah I, Jasmine L, Pushpavathi M (2012) Awareness of parents on the nature of cleft lip and palate: An exploratory study. JAIISH 30: 115-122.

22. Davis BL, MacNeilage PF (1995) The articulatory basis of babbling. J Speech Lang Hear Res 38(6): 1199-1211.

23. Willadsen E, Enemark H (2000) A comparative study of prespeech vocalizations in two groups of toddlers with cleft palate and a noncleft group. Cleft Palate Craniofac J 37(2): 172-178.

24. Chapman KL, Hardin MA (1991) Language input of mothers interacting with their young children with cleft lip and palate. Cleft Palate Craniofac J 28 (1): 78-85.
25. Scherer NJ, D Antonio L, McGahey H (2008) Early intervention for speech impairments in children with cleft palate. Cleft Palate Craniofac J 45: 18-31.

26. Scherer NJ (2001) Early vocalizations and later speech production in children with cleft lip and/or palate. Presented at the Ninth International Congress on Cleft Palate and Related Craniofacial Anomalies, Goteborg, Sweden.

27. Pamplona MC, Ysunza A, Uriostegui C (1996) Linguistic interaction: The active role of parents in speech therapy for cleft palate patients. Int J Pediatr Otorhinolaryngol 37: 17-27. 